\title{
Measuring menstrual hygiene experience: Development and validation of the Menstrual Practice Needs Scale (MPNS-36)
}

\author{
Julie Hennegan ${ }^{1}$ \\ Agnes Nansubuga ${ }^{2}$ \\ Calum Smith ${ }^{3}$ \\ Maggie Redshaw ${ }^{4}$ \\ Agnes Akullo ${ }^{2}$ \\ Kellogg J. Schwab ${ }^{1}$ \\ ${ }^{1}$ The Water Institute, Department of Environmental Health and Engineering, Johns Hopkins \\ Bloomberg School of Public Health, Baltimore, MD, USA \\ ${ }^{2}$ Irise Institute East Africa, Jinja, Uganda \\ ${ }^{3}$ Irise International, Sheffield, UK \\ ${ }^{4}$ Policy Research Unit in Maternal Health \& Care, National Perinatal Epidemiology Unit (NPEU), \\ University of Oxford, Oxford, UK
}

This is the preprint version of the manuscript.

The paper has now been published following peer-review.

Please view and cite the final version:

Hennegan, J., Nansubuga, A., Smith, C., Redshaw, M., Akullo, A., \& Schwab, K.J. (2020). Measuring menstrual hygiene experience: Development and validation of the Menstrual Practice Needs Scale (MPNS-36) in Soroti, Uganda. BMJ Open, 10, e034461. http://dx.doi.org/10.1136/bmjopen-2019034461

Correspondence:

Dr. Julie Hennegan

Johns Hopkins Bloomberg School of Public Health

Baltimore, MD, United States

jhenneg1@jhu.edu

Twitter: julie hennegan 


\begin{abstract}
Objectives. This study describes the development and validation the Menstrual Practice Needs Scale (MPNS-36) which measures the extent to which females' menstrual practices and environments meet their needs.
\end{abstract}

Methods. A 54-item pool was developed following systematic-review of qualitative and quantitative studies and expert feedback. Item reduction and scale validation were undertaken using a crosssectional survey of 538 menstruating schoolgirls in Soroti, Uganda. Test-retest reliability was assessed in a sub-sample of 52 girls two weeks after the first administration. Construct validity was tested through relationships with hypothesised correlates: confidence to manage menses, selfreported school absenteeism, and mental health symptoms.

Results. The final MPNS-36 comprises 28 items applicable to all respondents, and 8 items capturing washing and drying experiences for those reusing menstrual materials. A four-factor solution for the core 28 items was the best fit for the data (RMSEA=0.028-0.029; CFI=0.961-0.964; TFI=0.957-0.959), supplemented by two factors for reuse (RMSEA=0.021-0.030; $\mathrm{CFI}=0.987-0.994 ; \mathrm{TFI}=0.981-0.991$ ). Subscale and total scores were calculated as mean scores to support accessibility for practitioners. Subscales were 'material and home environment needs' (11 items, $\alpha_{\text {ordinal }}=0.84$ ), 'transport and school environment needs' (5-items, $\left.\alpha_{\text {ordinal }}=0.73\right)$, 'material reliability concerns' (3-items, $\left.\alpha_{\text {ordinal }}=0.55\right)$, 'change and disposal insecurity' (9-items, $\alpha_{\text {ordinal }}=0.80$ ), 'reuse needs' ( 5 -items, $\left.\alpha_{\text {ordinal }}=0.76\right)$, and 'reuse insecurity' ( 3 -items, $\alpha_{\text {ordinal }}=0.56$ ). Relationships between subscales and hypothesised correlates supported validity. Home- and school-based items were more strongly associated with confidence to manage menstruation at home and school, respectively. Higher scores predicted not missing school during the last menstrual period (total score: $\mathrm{OR}=2.62,95 \% \mathrm{Cl}=1.52$ 4.50). Test-retest reliability was moderate (total score: $\operatorname{ICC}_{(2,1)}=0.69$ ).

Conclusions. The MPNS-36 demonstrated acceptable reliability and validity. It is the first measure to capture women and girls perceived menstrual hygiene and may be used across a range of study designs to assess menstrual needs. Future research should explore the suitability and sensitivity of the measure across contexts. 


\section{INTRODUCTION}

Reports of women's and girls' negative experiences of menstruation have led to an increasing momentum to enact policies and programs to improve menstrual health.[1,2] A growing body of qualitative studies have described the challenges faced during menstruation and their implications for women's and girl's health and social participation.[3,4] Qualitative methods are well-suited to capturing the nuances of menstrual experience. However, quantitative studies are often needed to support decision making, evaluate interventions, and monitor progress. To date, quantitative studies have struggled to engage with the complexity of menstrual experiences and have been limited by the lack of available measures to capture core concepts.[5] Researchers have relied on study-based questionnaires in the absence of evidence to direct question selection or provide insights on measure reliability and validity.

This study reports on the development and validation of a new measure to capture women's and girls' perceptions of their menstrual management needs. Here we describe: the identification of the constructs targeted for assessment, the development of the Menstrual Practice Needs Scale (MPNS), and the pilot and validation of the measure in a sample of menstruating schoolgirls.

\section{Menstrual practice needs}

Establishing ways to measure menstrual hygiene has been an ongoing gap and research priority in the study of menstrual experience and interventions.[6-8] Good menstrual hygiene was initially defined as "women and adolescent girls using a clean menstrual management material to absorb or collect blood that can be changed in privacy as often as necessary for the duration of the menstruation period, using soap and water for washing the body as required, and having access to facilities to dispose of used menstrual management materials."[9] This highlighted females' physical management of menses.[10-12] The term has since seen new iterations, drawing in other menstrual needs including knowledge of the menstrual cycle and supportive socio-cultural environments free from stigma and menstrual-related restrictions.[12-14] To capture these varied aspects, multiple indicators with specific methods of assessment will be necessary. While the formal definitions of menstrual hygiene and menstrual health continue to evolve, the need for measures capturing the implicit core concepts remains unchanged.[8]

To inform our measure development efforts, we undertook a systematic review and meta-synthesis of extant qualitative studies of women's and girls' menstrual experiences in low- and middle-income countries (see [3]). We synthesised findings from 76 eligible studies to identify salient themes and their relationships, developing an integrated model of menstrual experience. Of the identified components of menstrual experience emerging from the review, two focused on women's and girls' physical management of menstrual bleeding; menstrual practices, and perceptions of menstrual practices and environments.[3] In describing the former, authors of included studies highlighted the range of practices undertaken to manage menses, often discussing the ways practices influenced discomfort or health. In the review we highlighted the distinction between these behavioural practices such as the type of material used, and individuals' perceptions of practices adequacy, comfort, or reliability. Perceptions reflected individual preferences and past experiences, resources, knowledge, expectations and the norms of their socio-cultural environments.

Quantitative study of menstrual experience has frequently collected data on individuals' menstrual practices.[7] We would argue that practices alone are not well placed to capture individuals' satisfaction or concerns, a frequent target for improvement in menstrual health programs. Measures assessing the type of material used do not reveal if this material was preferred, just as those 
capturing the quantity of materials used do not indicate if the user felt this was sufficient. Practices may be classified as more favourable based on their associations with reproductive tract infections,[15] but the usefulness of these categories is limited when considering program impacts on other outcomes such as menstrual experience, psychosocial wellbeing or social participation. We hypothesise that measures of individuals' perceived adequacy of practices and environments are likely to more closely align with findings from qualitative research and predict social participation and wellbeing, as they acknowledge that the same practices may be appraised differently due to a range of individual and sociocultural influences. We propose that quantitative assessment should include measures of women's perceptions along with their practices. Both approaches align with the existing description of menstrual hygiene, which does not specify whether adequate materials, disposal, cleanliness, or privacy are defined by investigators through top-down appraisal of behaviours or defined by respondents' perspectives.

Thus, in this study we aimed to develop a measure that can capture the extent to which respondents' current menstrual management practices and environments are perceived to meet their needs. We restrict the measure to the practices undertaken and environments used to manage menstrual bleeding; hypothesising that different measures will be needed to address other constructs relating to menstrual pain or knowledge which are outside the scope of this work. To test construct validity, we hypothesised that perceptions of menstrual practice needs would predict lower school absenteeism due to menstruation, higher confidence to manage menstruation, and fewer mental health symptoms, based on past qualitative research.[3]

\section{Measurement considerations}

MPNS development was informed by past research highlighting considerations for measurement and preliminary investigations by our study team. First, past research has indicated that poor attention to the full range of menstrual practices may provide a skewed appraisal of community needs.[16] Further, fixation on one menstrual practice in measurement, such as the type of material used, may lead to overemphasis on this aspect at the expense of others. The breadth of practices undertaken was informed through systematic review of past research.[3] Practices identified for the proposed measure were: menstrual materials used, frequency of changing materials, transportation and storage of materials, handwashing during menstrual management, genital and body cleaning, disposal of used materials, and methods of washing and drying materials, including access to a vessel for holding water and the use of soap. This list is consistent with an independent qualitative study which aimed to identify the breadth of practice challenges in India, lending further support to this broad coverage.[12] A second consideration was informed by a preliminary study investigating the location-dependency of menstrual practices. Through a cross-sectional study in Bangladesh, we found that schoolgirls' self-reported menstrual practices, such as the material used, varied between home and school environments, as did their confidence to manage menses. These findings suggest that self-report items with unclear locations may not adequately reflect the experiences researchers are aiming to measure.[17] Third, in focus group discussions (FGDs) with enumerators who had implemented Performance Monitoring and Accountability (2020) surveys [18] in Niger, participants reported that survey respondents rarely immediately understood the intention of items asking whether their menstrual environment was 'private' or 'safe'. Enumerators frequently provided clarifications based on their own understandings; which also differed. Findings from FGDs suggested that 'privacy' and 'safety' as stand-alone terms may not be amenable to cross-cultural adaptation and translation. Similar issues with the interpretation of 'privacy' were reported in an independent field test of measures in Belize.[19] For questions aimed at capturing these concepts, we returned to the qualitative studies from which they were drawn and identified worries about being seen or 
harmed as origins of 'privacy' and 'safety' priorities. This approach aligned with a recent measure of sanitation insecurity.[20] Finally, practitioners and researchers alike recognise the sometimes contradictory requirements in wishing to best capture experiences and at the same time moderate participant fatigue and survey length. Thus, the measure needed to balance length with comprehensiveness.

In sum, grounded in past research we defined menstrual practice needs as a core construct for measurement, and drew on past studies and preliminary research to guide item development.

\section{METHODS}

The MPNS was developed across three phases, summarised in Figure 1.

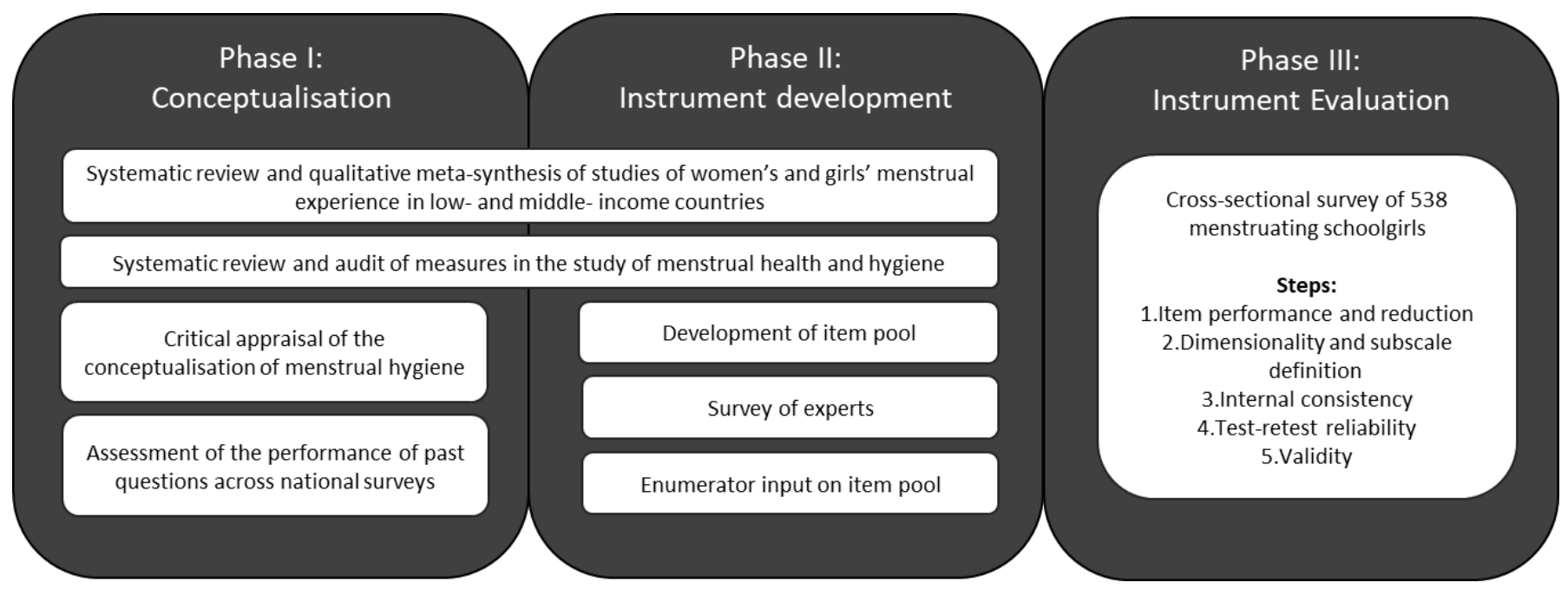

Figure 1. Phases of development of the MPNS-36

\section{Conceptualisation}

In the first phase we identified constructs for assessment through systematic reviews of past research, assessed the need for new measures, and collated insights from the performance of past questions. This is described in the introduction.

\section{Instrument development}

Using our systematic review of qualitative studies, we collated the menstrual practices reported, and illustrative quotations of participants' perceptions of their practices and environments. These were included in the meta-synthesis report, see [3]. We also utilised the full set of studies thematically coded in Nvivo 12 during the review, to provide an extensive set of quotations from which to draw scale items.

Following initial item generation, we undertook an online survey of experts. We invited members of the East and Southern Africa Menstrual Hygiene Research Network, and experts attending past MHM in Ten [21] meetings to participate. Twenty-three experts provided feedback on a selection of 19 MPNS draft items. Participants identified as researchers (52\%), practitioners (12\%), or both (36\%). Experts rated the usefulness of MPNS items and were invited to make comments. One item was removed from the pool due to poor ratings. Experts were also consulted regarding the response 
format with $68 \%$ endorsing a 4-point Likert option. A further $14 \%$ preferred a 3-point scale, with others suggesting dichotomous responses or responses varied by context/language.

Sixteen items, professionally translated into French, were presented to resident enumerators following collection of Performance Monitoring and Accountability (PMA) 2020 surveys in Niamey, Niger. Items were presented as part of focus group discussions (FGDs) concerning the performance of menstrual hygiene questions in PMA2020 surveys. Twenty resident enumerators from Niamey provided feedback on the response options, with endorsement of a 4-point scale. During FGDs enumerators indicated two potentially problematic items, suggesting that these were less likely to be reported honestly by older adult women. These items were removed after piloting. During FGDs, enumerators were asked for their impressions of what each item sought to capture. Their interpretations matched our intentions for the items, supporting face validity.

Final item wording was refined during translation and back-translation of items and research assistant training for the validation study in Uganda.

\section{Instrument evaluation: Study sample and data collection}

The target sample size was based on ten participants per item, a 10:1 ratio. A cross-sectional survey was undertaken across 12 schools in Soroti, Uganda. Soroti is a regional urban centre in the Teso sub-region of Eastern Uganda. Ugandan Demographic Household Survey (DHS) data from 2016 reports that $41.5 \%$ of the Teso region population places in the lowest national wealth quintile. According to DHS, $39.2 \%$ of households had an observed hand washing location, $63.7 \%$ of females had attended some primary school as their highest educational attainment.[22]

Schools recruited for the survey were already engaged with the partner NGO, Irise Institute East Africa, were all government schools, and had been selected by the District Education Office as those with the greatest need. Data were collected March-May 2019. Girls 12 years and older were recruited from Primary (P) class levels P5-6, with expansion to P4 and P7 to achieve the required sample size. In the previous year (October 2018), pupils in P6 received a menstrual education and product (reusable sanitary pad) intervention. These students should have graduated to P7 by the time of the survey. Grade repetition, school transfer, and the inclusion of some P7 students to achieve the required sample meant some participants in this study had received an intervention 5-6 months prior to the survey.

Female research assistants, local to the area were trained to deliver the survey. Paper surveys in English were self-completed by girls in groups of no more than six, with research assistants providing verbal instruction and translation in Ateso. Group surveys lasted approximately 75-90 minutes.

Girls needed to be present at school and were recruited by class. If more girls were available than could be surveyed, participants were selected using a simple randomisation technique (every third girl across desk rows, repeated until the maximum number was met). Schools had at least two visits for data collection. Almost all menstruating girls in participating classes were sampled to achieve the target sample size. During the first data collection visit in the first 10 schools, one pre-selected research assistant consented her group of up to 6 girls for re-test survey and recorded their names next to an ID number. A reserve group of girls were also consented. Upon repeat visit, the target retest group were sought, with substitutions from the reserve group if needed.

Data were entered into Qualtrics survey system by trained research assistants. Fifty surveys, 9.29\%, were entered twice for error screening. Data entry error rate was $1.59 \%$. 


\section{Survey content and question format}

Demographics. Participants self-reported their age, class level, religion and grade repetition. Household resources were assessed using four items from the Afrobarometer lived poverty index,[23] indicating how often, over the past year, girls went without food, clean water, medicine and school supplies.

Menstrual practices. A suite of questions asking girls about their menstrual practices were asked as part of the concurrent development of the menstrual practices questionnaire (MPQ). Behaviours were reported for the last menstrual period, consistent with MPNS items. For the present study, we used items capturing the menstrual materials used during the last period at home, frequency of change of menstrual materials and location of material change.

Last menstruation. We asked girls to estimate the timing of their last menstrual period in broad terms "I have my period now", "last week", "within two weeks", "within three weeks", "one month" or "more than one month ago". For girls undertaking the re-test survey, those selecting the first two options were coded as reporting on a new period.

MPNS item pool. The $54 \mathrm{draft}$ items were included in the participant survey. On each page, participants were reminded that items referred to the last menstrual period. The items took the form of a personal statement followed by response options "never", "sometimes", "often", and "always". Response options were accompanied by a visual tool, see Figure 2. Participants had been familiarized with Likert responding earlier in the survey for agreement and disagreement items. The MPNS section of the survey was preceded by an activity. Research assistants had a large version of the visual tool and asked participants to report as a group on the frequency of a variety of school activities. For example, "How often do you have a lunch break during the school day?", "How often do you have tests at school?". The activity allowed research assistants to engage students regarding the selected response category.

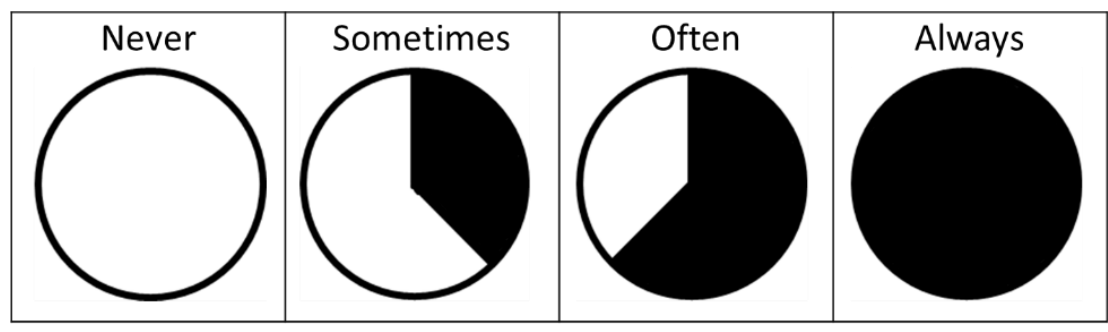

Figure 2. Visual chart for MPNS item response options

Psychological symptoms. Psychological health was assessed using a modified version of the Depression, Anxiety, Stress Scale (DASS-21).[24] For length, we selected only the depression and anxiety subscales and one item was removed from each scale. We removed anxiety item "I was aware of dryness of my mouth", and depression item "I felt down-hearted and blue" as these were perceived to present challenges for translation. Language was simplified for translation, for example "I couldn't seem to experience any positive feeling at all" was simplified to "I couldn't experience any positive feelings". Participants reported how often over the past week they experienced each statement in the list. Response options were simplified to: 0 "never", 1 "sometimes", 2 "often", 3 "almost always". For analysis we used a total symptoms score, with depression and anxiety items transposed onto a 7-point scale to reflect the original. Total scores could range from 0 to 42. 
Confidence to manage menstruation. Girls reported on a 4-point Likert scale from "Strongly disagree" to "Strongly agree" their agreement with the statement "I feel confident to manage my menstrual period at home" and "at school". This was accompanied with a note that managing menstruation means "collecting materials, changing, washing drying and disposing of materials during your period". Dichotomous responses of "confident" (agree or strongly agree) and "not confident" (disagree or strongly disagree) were used for analyses.

School attendance. Participants self-reported if they "usually" missed school during menstruation, providing "yes" or "no" responses. For comparison, girls reported if they missed school during their last menstrual period "yes", "no", or "not applicable" if their last period did not fall during school time.

\section{Instrument evaluation: Analyses}

Analyses were undertaken using Stata 15 and R Version 3.6.0.

Item responses were investigated through descriptive statistics. We used random split-halves of the data to develop then test the emerging factor structure. Acknowledging the ordinal nature of the data, exploratory factor analysis (EFA) with principal axis factoring was undertaken using the polychoric correlation matrix. We used scree plots, eigenvalues $>1$ (Kaiser criterion), and theoretical plausibility as criteria against which item reduction and final factor structure were determined. We anticipated a-priori that any emergent factors would be corelated, and specified oblique rotation, using promax with Kaiser normalisation. To maintain content validity, we prioritised coverage of menstrual practices before selecting items with the highest factor loadings during reduction. Items with loadings $<0.30$ were considered to have poor loading. During exploratory factor analysis we permitted cross-loading for two items which applied to both school and home settings. These were confined to a single factor in the final model.

We explored potential predictors of missing data including class level, age, and household resources and identified no pattern of missing data. Little's MCAR (missing completely at random) test was non-significant $\chi^{2}=4107.57, p=0.246$, further supporting our assumption that there was no pattern. A total of 13 girls (2.4\%) were missing more than 2 items on the final 28-core item measure and were excluded from final analysis. Missing data were deleted pairwise for EFAs.

We undertook confirmatory factor analysis (CFA) using the lavaan package in R. Reflecting the ordinal nature of the data, we used a robust diagonally weighted least squares estimator (DWLS). DWLS requires complete data. Multiple imputation using chained equations with the mice package in $\mathrm{R}$ was undertaken for girls with 2 or fewer missing items. As lavaan does not support multiply imputed data with DWLS estimation, we extracted ten imputed data sets and ran the CFA on each. We combined factor loadings using Rubin's rules.[25] There is little guidance on combining model fit statistics across imputations, so we provide the range of root mean square error of approximation (RMSEA), comparative fit index (CFI) and Tucker-Lewis index (TLI). [26] We considered RMSEA $\leq 0.05$ as indicative of close fit, with RMSEA $\leq 0.08$ as fair fit, and CFI and TFI $\geq 0.95$ as indicative of acceptable model fit.(27) Final CFA structure was compared to bi-factor and hierarchical models using model fit statistics, item loadings and theoretical plausibility.

Measurement invariance was assessed by comparing the final CFA model between girls' who reported using only disposable sanitary pads at home, to others. We tested for threshold and loading invariance, using updated guidance for multi-group CFA for ordinal data.[28,29] 
Internal consistency was computed using the polychoric, rather than Pearson, correlation matrix to generate an ordinal alpha.[30] We also provided Cronbach's alpha based on Pearson's correlations for comparison, although this has been suggested to underestimate associations in ordinal data.(30) We prioritised capturing experiences across the breadth of menstrual practices, recognising that measurement can bias attention towards particular practices. We also hypothesised that girls were likely to experience varied practices and environments with different levels of acceptability. Thus, apriori, we were willing to sacrifice some degree of internal consistency for coverage. Nevertheless, we applied a conventional $\alpha_{\text {ordinal }} \geq 0.70$ as indicative of satisfactory reliability. Test-retest reliability was assessed using intra class correlation coefficients (ICCs) calculated using single-measure, twoway mixed-effects models, with absolute agreement.[31] We assessed test-retest reliability separately for girls reporting on the same or different menstrual period to their original survey. Although guidelines on acceptable ICCS are unclear, we considered an ICC between 0.50 and 0.75 to represent moderate reliability, and greater than 0.75 to represent good reliability.[31]

The lack of available measures for menstrual health constructs limited comparators for convergent or divergent validity. Drawing on hypotheses from qualitative research, we tested predictive validity though associations between the MPNS and confidence to manage menstruation, mental health and school absenteeism. Bivariate relationships were tested using Pearson's correlation coefficients for continuous variables, and binary logistic regressions for dichotomous outcomes (school absenteeism, and confidence to manage menstruation).

\section{RESULTS}

\section{Participants}

A total of 538 menstruating girls were surveyed. The mean age of the sample was 14.49 ( $S D=1.20)$. Self-reported ages were 12-19, with one girl indicating 11 years on the survey but reporting being 12 during eligibility screening. Most of the sample were drawn from Primary Class Level (P)6, 59.29\%, with an additional $18.40 \%$ from P5, 16.91\% from P7 and 5.39\% from P4. Most girls, $72.95 \%$, had repeated a class level. Ninety-five per cent of the population were Christians with the remaining $5 \%$ Muslim. Of the sample, $83.07 \%$ had gone without food, water, medicine or school supplies in the past year. The mean score for symptoms using DASS-21 items was 12.66 (SD=6.48).

In multi-response option questions capturing all menstrual materials used at home during the last menstrual period: $58.10 \%$ of girls used disposable pads, $32.03 \%$ reusable pads, $19.93 \%$ cloth, $13.22 \%$ used their underwear alone, $7.64 \%$ toilet paper, $7.26 \%$ cotton wool and $5.40 \%$ used mattress and other materials. A total of 291 girls (54.49\%) washed and reused menstrual materials during their last period.

A total of $59.14 \%$ changed materials three or more times on their heaviest day. Materials were changed in a bedroom (52.42\%), a bathroom (26.39\%), latrine $(19.89 \%)$ or outside $(1.30 \%)$ when at home. Most girls, $87.71 \%$, had changed materials away from home at least one day during their last period.

\section{Item responses}

The proportions of responses, and number of missing, for each item in the 54-item pool are presented in Table 1. Frequencies highlight the menstrual management challenges facing girls. They also show a lower proportion of girls using the 'often' response option. There was a low proportion of missing data across scale items, varying from $0.00 \%-4.46 \%$. 


\section{Item reduction}

We removed items fitting poorly with a parsimonious and theoretically plausible factor structure, and with the objective of balancing length with coverage. This meant poorly loading items, and some items that duplicated concepts and had high intercorrelations were removed. Excluded items, with reasons, are presented in Supplementary Materials 1.

Notably for item reduction, only $27 \%$ of girls always felt comfortable to use the same location for urination during their period as when they were not menstruating, with a lower $23 \%$ girls comfortable at school (items 33 and 37). This casts some doubts regarding responses to the subsequent items, item 34 and 38, wherein girls reported their worries that others would see their menstrual blood after urination. It is unclear if this question can apply accurately to those who may have avoided usual latrines during menstruation. In EFAs we found items 33 and 37, and items 34 and 38 loaded on their own factors. Two-item factors were not considered acceptable for the measure and all four items were excluded.[32] 
Table 1. Full 54 item pool and participant responding $(n=538)$

\begin{tabular}{|c|c|c|c|c|c|c|}
\hline No. & Item & $\begin{array}{l}\text { Never } \\
\%\end{array}$ & $\begin{array}{l}\text { Some- } \\
\text { times \% }\end{array}$ & $\begin{array}{l}\text { Often } \\
\%\end{array}$ & $\begin{array}{l}\text { Always } \\
\%\end{array}$ & $\begin{array}{l}\text { Missing } \\
\text { (n) }\end{array}$ \\
\hline 1 & I was able to choose the menstrual materials I most wanted to use & 14.95 & 39.44 & 6.92 & 38.69 & 3 \\
\hline 2 & My menstrual materials were comfortable & 14.53 & 29.62 & 11.32 & 44.53 & 8 \\
\hline 3 & $\begin{array}{l}\text { (r) I worried that my menstrual materials would allow blood to pass } \\
\text { through to my outer garments }\end{array}$ & 28.63 & 38.42 & 14.69 & 18.27 & 7 \\
\hline 4 & $\begin{array}{l}\text { (r) I worried that my menstrual materials would move from place } \\
\text { while I was wearing them }\end{array}$ & 30.92 & 44.08 & 9.92 & 15.08 & 14 \\
\hline 5 & $\begin{array}{l}\text { (r) I worried about how I would get more of my menstrual material if I } \\
\text { ran out }\end{array}$ & 23.56 & 43.87 & 13.41 & 19.16 & 16 \\
\hline 6 & $\begin{array}{l}\text { I had enough of my menstrual materials to change them as often as I } \\
\text { wanted to }\end{array}$ & 18.73 & 33.59 & 11.58 & 36.10 & 20 \\
\hline 7 & I was satisfied with the cleanliness of my menstrual materials & 13.42 & 23.15 & 13.04 & 50.39 & 24 \\
\hline 8 & I could get more of my menstrual materials when I needed to & 19.35 & 41.18 & 13.09 & 26.38 & 11 \\
\hline 9 & $\begin{array}{l}\text { I felt comfortable carrying spare menstrual materials with me outside } \\
\text { my home }\end{array}$ & 30.86 & 32.76 & 10.86 & 25.52 & 13 \\
\hline 10 & $\begin{array}{l}\text { I felt comfortable carrying menstrual materials to the place where I } \\
\text { changed them }\end{array}$ & 30.12 & 30.69 & 11.39 & 27.80 & 20 \\
\hline 11 & $\begin{array}{l}\text { I felt comfortable transporting used materials to wash or dispose of } \\
\text { them }\end{array}$ & 22.81 & 31.18 & 12.74 & 33.27 & 12 \\
\hline 12 & $\begin{array}{l}\text { I had a clean place to store my menstrual materials when I was not } \\
\text { using them during my period }\end{array}$ & 12.69 & 23.11 & 10.42 & 53.79 & 10 \\
\hline 13 & $\begin{array}{l}\text { (r) I was worried that someone would see my stored menstrual } \\
\text { materials when I was not using them }\end{array}$ & 24.95 & 44.47 & 10.32 & 20.26 & 5 \\
\hline 14 & I felt comfortable storing my menstrual materials until my next period & 14.66 & 21.80 & 7.52 & 56.02 & 6 \\
\hline 15 & I was able to wash my hands when I wanted to & 4.31 & 24.72 & 6.37 & 64.61 & 4 \\
\hline 16 & I was able to wash my vagina when I wanted to & 10.53 & 16.35 & 10.71 & 62.41 & 6 \\
\hline 17 & I was able to wash my hands and vagina as often as I wanted to & 9.33 & 20.19 & 12.00 & 58.48 & 13 \\
\hline 18 & $\begin{array}{l}\text { (r) I was concerned that I would not have enough soap to wash my } \\
\text { hands or vagina }\end{array}$ & 19.77 & 46.90 & 12.98 & 20.35 & 22 \\
\hline 19 & I felt clean during my last period & 13.02 & 25.09 & 13.09 & 47.92 & 8 \\
\hline 20 & (r) I worried about where to dispose of my used menstrual materials & 36.55 & 30.49 & 11.93 & 21.02 & 10 \\
\hline 21 & $\begin{array}{l}\text { (r) I worried that people, or animals, may be able to access my used } \\
\text { menstrual materials after I disposed of them }\end{array}$ & 44.03 & 30.22 & 9.89 & 15.86 & 2 \\
\hline 22 & $\begin{array}{l}\text { (r) I was concerned that others would see my used menstrual } \\
\text { materials in the place I disposed of them }\end{array}$ & 33.02 & 38.81 & 12.31 & 15.86 & 2 \\
\hline 23 & I was able to immediately dispose of my used menstrual materials & 15.46 & 26.07 & 11.73 & 46.74 & 1 \\
\hline 24 & I was able to dispose of my used materials in the way that I wanted to & 21.00 & 19.89 & 11.90 & 47.21 & 0 \\
\hline 25 & $\begin{array}{l}\text { When at home, I was able to change my menstrual materials when I } \\
\text { wanted to }\end{array}$ & 6.16 & 23.13 & 6.16 & 64.55 & 2 \\
\hline 26 & $\begin{array}{l}\text { When at home, I was satisfied with the place I used to change my } \\
\text { menstrual materials }\end{array}$ & 8.07 & 23.45 & 12.38 & 56.10 & 5 \\
\hline 27 & When at home, I had a clean place to change my menstrual materials & 5.69 & 18.41 & 11.76 & 64.14 & 11 \\
\hline 28 & $\begin{array}{l}\text { (r) When at home, I worried that I would not be able to change my } \\
\text { menstrual materials when I needed to }\end{array}$ & 30.22 & 35.63 & 14.93 & 19.22 & 2 \\
\hline 29 & $\begin{array}{l}\text { (r) When at home, I worried that someone would see me while I was } \\
\text { changing my menstrual materials }\end{array}$ & 30.58 & 41.65 & 8.82 & 18.95 & 5 \\
\hline 30 & $\begin{array}{l}\text { (r) When at home, I worried that someone would harm me while I was } \\
\text { changing my menstrual materials }\end{array}$ & 46.60 & 26.76 & 9.81 & 16.79 & 8 \\
\hline 31 & $\begin{array}{l}\text { (r) When at home, I worried that something else would harm me } \\
\text { while I was changing my menstrual materials (e.g., animals, insects, } \\
\text { unsafe structure) }\end{array}$ & 47.96 & 32.34 & 8.36 & 11.34 & 0 \\
\hline
\end{tabular}




\begin{tabular}{|c|c|c|c|c|c|c|}
\hline 32 & $\begin{array}{l}\text { (r) When at home, I worried that someone would see me when I was } \\
\text { washing my vagina }\end{array}$ & 39.25 & 35.85 & 8.49 & 16.42 & 8 \\
\hline 33 & $\begin{array}{l}\text { When at home, I felt comfortable using the same location for } \\
\text { urination as when I do not have my period }\end{array}$ & 37.27 & 25.84 & 9.93 & 26.97 & 4 \\
\hline 34 & $\begin{array}{l}\text { (r) When at home, I worried that others would see my menstrual } \\
\text { blood after I had urinated }\end{array}$ & 29.66 & 38.43 & 9.70 & 22.20 & 2 \\
\hline 35 & $\begin{array}{l}\text { When at school, I was able to change my menstrual materials when I } \\
\text { wanted to }\end{array}$ & 34.51 & 33.77 & 6.72 & 25.00 & 2 \\
\hline 36 & $\begin{array}{l}\text { When at school, I was satisfied with the place I used to change my } \\
\text { menstrual materials }\end{array}$ & 29.06 & 30.19 & 12.08 & 28.68 & 8 \\
\hline 37 & $\begin{array}{l}\text { When at school, I felt comfortable using the same location for } \\
\text { urination as when I do not have my period }\end{array}$ & 38.97 & 28.25 & 9.60 & 23.35 & 7 \\
\hline 38 & $\begin{array}{l}\text { (r) When at school, I worried that others would see my menstrual } \\
\text { blood after I had urinated }\end{array}$ & 28.89 & 39.77 & 10.69 & 20.64 & 5 \\
\hline 39 & When at school, I had a clean place to change my menstrual materials & 30.17 & 26.94 & 10.44 & 32.45 & 11 \\
\hline 40 & $\begin{array}{l}\text { (r) When at school, I worried that I would not be able to change my } \\
\text { menstrual materials when I needed to }\end{array}$ & 23.21 & 41.51 & 13.02 & 22.26 & 8 \\
\hline 41 & $\begin{array}{l}\text { (r) When at school, I worried that someone would see me while I was } \\
\text { changing my menstrual materials }\end{array}$ & 28.63 & 39.55 & 12.99 & 18.83 & 7 \\
\hline 42 & $\begin{array}{l}\text { (r) When at school, I worried that someone would harm me while I } \\
\text { was changing my menstrual materials }\end{array}$ & 37.83 & 30.90 & 10.67 & 20.60 & 4 \\
\hline & Items relevant to those washing and reusing materials $(n=291)$ & & & & & \\
\hline 43 & I had enough water to soak or wash my menstrual material & 6.23 & 20.76 & 5.54 & 67.47 & 2 \\
\hline 44 & $\begin{array}{l}\text { I had access to a basin to soak or wash my menstrual materials } \\
\text { whenever I needed it }\end{array}$ & 13.06 & 26.12 & 9.97 & 50.86 & 0 \\
\hline 45 & I was able to wash my menstrual materials when I wanted to & 15.14 & 23.94 & 7.75 & 53.17 & 7 \\
\hline 46 & I had enough soap to wash my menstrual materials & 8.80 & 31.34 & 9.51 & 50.35 & 7 \\
\hline 47 & $\begin{array}{l}\text { (r) I worried that someone would see me while I was washing my } \\
\text { menstrual materials }\end{array}$ & 27.92 & 42.76 & 12.01 & 17.31 & 8 \\
\hline 48 & $\begin{array}{l}\text { (r) I worried about how I would get soap to wash my menstrual } \\
\text { materials }\end{array}$ & 31.07 & 38.93 & 12.14 & 17.86 & 11 \\
\hline 49 & $\begin{array}{l}\text { (r) I worried that my menstrual materials would not be dry when I } \\
\text { needed them }\end{array}$ & 31.49 & 39.10 & 13.49 & 15.92 & 2 \\
\hline 50 & $\begin{array}{l}\text { (r) I worried that others would see my menstrual materials while they } \\
\text { were drying }\end{array}$ & 23.08 & 42.31 & 12.24 & 22.38 & 5 \\
\hline 51 & I was able to dry my materials when I wanted to & 12.98 & 22.11 & 13.33 & 51.58 & 6 \\
\hline 52 & I was satisfied with the place I used to dry my menstrual materials & 15.03 & 25.87 & 9.44 & 49.65 & 5 \\
\hline 53 & $\begin{array}{l}\text { I was satisfied with the appearance of my menstrual materials after I } \\
\text { had cleaned them }\end{array}$ & 8.04 & 24.83 & 12.24 & 54.90 & 5 \\
\hline 54 & $\begin{array}{l}\text { I was satisfied with the smell of my menstrual materials after I had } \\
\text { cleaned them }\end{array}$ & 21.80 & 25.26 & 7.27 & 45.67 & 2 \\
\hline
\end{tabular}

(r) reverse scored. 


\section{Dimensionality}

EFA on the first random split-half of the data was undertaken, first for the items applying to all respondents. This process concluded with a 28 -item, four-factor solution explaining $80 \%$ of the total variance. Factorability was confirmed through visual inspection of the polychoric correlation matrix, and Kaiser-Meyer-Olkin (KMO) sampling adequacy of 0.72 for the final 28 -item split-half sample. Thirteen girls were missing more than two items on the final 28 core items that applied to all respondents. These participants were excluded from subsequent analyses.

A separate EFA was undertaken in the sub-sample of participants who reported they had washed and reused materials during their last period and answered questions concerning washing and drying during the last period $(n=286)$. A two-factor solution was retained, with a total of eight items of the original 12. Factor structure and loadings are presented in Table 2.

EFA was followed by a CFA of the second split-half of the data for the 28 core items, and the entire subset of those reusing materials for the additional 8 reuse items. The four-factor model was a good fit for the data (RMSEA=0.028-0.029; $\mathrm{CFI}=0.961-0.964 ; \mathrm{TFI}=0.957-0.959$ ). In the initial EFA solution two items ( 9 and 10) were cross loaded on home and school-related domains. This fit theoretically with the data since these items did not specify a location. In CFA on the second split-half, these items loaded more strongly on the school-factor and loaded poorly on the home-factor. These items were retained under only the 'transport and school environment needs' factor. A final CFA on the full data set supported good model fit for the core 28-items (RMSEA $=0.028-0.029 ; \mathrm{CFI}=0.957-0.959$; $\mathrm{TFI}=0.953-0.955$ ), and the additional reuse items (RMSEA=0.021-0.030; $\mathrm{CFI}=0.987-0.994 ; \mathrm{TFI}=0.981-$ $0.991)$.

The CFA on the full data set was compared to bi-factor and hierarchical models using structural equation models (SEM). Neither a bi-factor (RMSEA $=0.041 ; \mathrm{CFI}=0.913 ; \mathrm{TFI}=0.906$ ) nor a hierarchical model (RMSEA $=0.051 ; \mathrm{CFI}=0.877 ; \mathrm{TFI}=0.855$ ) were a better fit for the first imputed data set and were not investigated further.

Model invariance in the full data set was assessed, comparing those exclusively using disposable sanitary pad $(n=191)$ to others $(n=334)$. A model constraining both thresholds and loadings remained an acceptable fit (RMSEA=0.029; $\mathrm{CFI}=0.948 ; \mathrm{TFI}=0.947$ ) supporting the generalisation of latent constructs (subscales) across these two groups and suggesting that scores can be meaningfully compared across those using different menstrual materials. Item 6 , having enough materials to change as often as desired, loaded more poorly when groups were separated (estimate $=0.36$ ) which may indicate some variability in this question based on material type. 
Table 2. Factor structure and loadings for split-half EFA, CFA, and full sample CFA

\begin{tabular}{|c|c|c|c|}
\hline Item Number & $\begin{array}{l}\text { Split-half } \\
\text { EFA (n=261) }\end{array}$ & $\begin{array}{l}\text { Split-half } \\
\text { CFA }(n=264)\end{array}$ & $\begin{array}{l}\text { Full sample } \\
\text { Final CFA } \\
(n=525)\end{array}$ \\
\hline \multicolumn{4}{|c|}{ Material and home environment needs } \\
\hline 2 & 0.46 & 0.62 & 0.53 \\
\hline 6 & 0.45 & 0.52 & 0.45 \\
\hline 7 & 0.62 & 0.58 & 0.62 \\
\hline 8 & 0.38 & 0.54 & 0.49 \\
\hline 9 & 0.36 & -0.03 & - \\
\hline 10 & 0.36 & 0.11 & - \\
\hline 14 & 0.71 & 0.41 & 0.53 \\
\hline 15 & 0.64 & 0.64 & 0.59 \\
\hline 23 & 0.52 & 0.54 & 0.58 \\
\hline 24 & 0.43 & 0.61 & 0.56 \\
\hline 25 & 0.75 & 0.62 & 0.67 \\
\hline 26 & 0.61 & 0.68 & 0.67 \\
\hline 27 & 0.71 & 0.60 & 0.65 \\
\hline \multicolumn{4}{|c|}{ Transport and school environment needs } \\
\hline 9 & 0.27 & 0.63 & 0.58 \\
\hline 10 & 0.25 & 0.47 & 0.58 \\
\hline 35 & 0.55 & 0.60 & 0.60 \\
\hline 36 & 0.65 & 0.63 & 0.62 \\
\hline 39 & 0.76 & 0.66 & 0.64 \\
\hline \multicolumn{4}{|c|}{ Material reliability concerns } \\
\hline 3 & 0.63 & 0.51 & 0.53 \\
\hline 4 & 0.31 & 0.51 & 0.54 \\
\hline 5 & 0.57 & 0.55 & 0.52 \\
\hline \multicolumn{4}{|c|}{ Change and disposal insecurity } \\
\hline 20 & 0.40 & 0.49 & 0.48 \\
\hline 22 & 0.49 & 0.61 & 0.57 \\
\hline 28 & 0.47 & 0.50 & 0.50 \\
\hline 29 & 0.31 & 0.54 & 0.52 \\
\hline 30 & 0.72 & 0.78 & 0.72 \\
\hline 31 & 0.50 & 0.66 & 0.64 \\
\hline 40 & 0.62 & 0.46 & 0.48 \\
\hline 41 & 0.54 & 0.51 & 0.53 \\
\hline 42 & 0.54 & 0.66 & 0.57 \\
\hline $\begin{array}{l}\text { Reuse items } \\
\text { Item number }\end{array}$ & $\begin{array}{l}\text { Full-sample } \\
\text { EFA }(n=286)\end{array}$ & (N/A) & $\begin{array}{l}\text { Full sample } \\
\text { CFA }(n=286)\end{array}$ \\
\hline \multicolumn{4}{|l|}{ Reuse needs } \\
\hline 43 & 0.71 & - & 0.74 \\
\hline 44 & 0.53 & - & 0.53 \\
\hline 45 & 0.58 & - & 0.59 \\
\hline 46 & 0.66 & - & 0.68 \\
\hline 51 & 0.57 & - & 0.58 \\
\hline \multicolumn{4}{|c|}{ Reuse insecurity } \\
\hline 47 & 0.57 & - & 0.69 \\
\hline 49 & 0.42 & - & 0.45 \\
\hline 50 & 0.54 & - & 0.53 \\
\hline
\end{tabular}




\section{Scale scores and reliability}

Subscale scores and total score were calculated as mean scores where never $=0$, sometimes $=1$, often $=2$ and always $=3$ for positively coded items, and the reverse for negatively coded items. All subscales have ranges from 0 to 3 , and higher scores represent more positive experiences. Subscales specific to those reusing materials were only calculated for this population. Total score included reuse items for those to whom these were applicable. Plots showing relationships between the core four factors, and the total score are displayed in Supplementary Materials 2.

Cronbach's $\alpha$, and ordinal $\alpha$ are presented in Table 3. Acceptable reliability was achieved for most subscales. The two three-item subscales, material concerns and reuse insecurity, had poorer internal consistency.

Fifty-six girls completed the re-test survey. Of those, three were missing scores on MPNS items at original survey, and one had more than two missing items on the retest. Test-retest reliability for the 52 participants with repeat data using single-measure ICC are displayed in Table 3 . Reliability varied meaningfully between girls we estimated to be reporting on the same menstrual period as the original survey, compared to those who reported having a new period. We took the reliability among the subsample of girls reporting on the same menstrual period $(n=20)$ as indicative of scale reliability as questions specifically ask about the last period. 
Table 3. Scale scores, internal consistency, test-retest reliability, and interrelationships

\begin{tabular}{|c|c|c|c|c|c|c|c|c|c|c|c|}
\hline & \multirow[b]{2}{*}{ Mean (SD) } & \multicolumn{2}{|c|}{ Internal Consistency } & \multicolumn{2}{|c|}{$\begin{array}{l}\text { Test-retest reliability } \operatorname{ICC}_{(2,1)} \\
\qquad(95 \% \mathrm{Cl})\end{array}$} & \multicolumn{6}{|c|}{ Correlations between subscales (Pearson's $r$ ) } \\
\hline & & $\alpha$ & $\begin{array}{c}\text { Ordinal } \\
\alpha\end{array}$ & $\begin{array}{l}\text { Same period } \\
(n=20)\end{array}$ & $\begin{array}{c}\text { New period } \\
(n=32)\end{array}$ & 1 & 2 & 3 & 4 & 5 & 6 \\
\hline Total score $(n=525)$ & $1.82(0.37)$ & 0.77 & 0.82 & $\begin{array}{c}0.69 \\
(0.36-0.86)\end{array}$ & $\begin{array}{c}0.30 \\
(-0.05-0.58)\end{array}$ & 0.75 & 0.54 & 0.31 & 0.44 & 0.51 & 0.30 \\
\hline $\begin{array}{l}\text { 1. Material and home } \\
\text { environment needs }\end{array}$ & $1.99(0.62)$ & 0.79 & 0.84 & $\begin{array}{c}0.53 \\
(0.12-0.78)\end{array}$ & $\begin{array}{c}0.46 \\
(0.14-0.69)\end{array}$ & 1.00 & 0.51 & -0.08 & -0.15 & 0.65 & -0.02 \\
\hline $\begin{array}{l}\text { 2. Transport and school } \\
\text { environment needs }\end{array}$ & $1.35(0.78)$ & 0.66 & 0.73 & $\begin{array}{c}0.67 \\
(0.33-0.85)\end{array}$ & $\begin{array}{c}0.22 \\
(-0.14-0.53)\end{array}$ & - & 1.00 & -0.20 & -0.24 & 0.43 & -0.09 \\
\hline 3. Material reliability concerns & $1.81(0.73)$ & 0.51 & 0.55 & $\begin{array}{c}0.24 \\
(-0.22-0.61) \\
\end{array}$ & $\begin{array}{c}0.08 \\
(-0.27-0.41)\end{array}$ & - & - & 1.00 & 0.43 & -0.01 & 0.35 \\
\hline 4. Change and disposal insecurity & $1.87(0.61)$ & 0.74 & 0.80 & $\begin{array}{c}0.56 \\
(0.17-0.80) \\
\end{array}$ & $\begin{array}{c}0.16 \\
(-0.20-0.48) \\
\end{array}$ & - & - & - & 1.00 & -0.15 & 0.50 \\
\hline 5. Reuse needs $(n=286)$ & $2.08(0.72)$ & 0.66 & 0.76 & $\begin{array}{c}n=12 \\
0.67 \\
(0.19-0.89)\end{array}$ & $\begin{array}{c}n=17 \\
0.72 \\
(0.38-0.89)\end{array}$ & - & - & - & - & 1.00 & 0.06 \\
\hline 6. Reuse insecurity $(n=286)$ & $1.78(0.73)$ & 0.47 & 0.56 & $\begin{array}{c}-0.07 \\
(-0.60-0.50)\end{array}$ & $\begin{array}{c}0.23 \\
(-0.26-0.63)\end{array}$ & - & - & - & - & - & 1.00 \\
\hline
\end{tabular}




\section{Validity}

Content validity of the scale was assessed through comparison with findings from qualitative research, FGDs with enumerators undertaking surveys of menstrual hygiene, and survey of experts.

For construct validity, we tested predictive relationships between scale scores and confidence to manage menstruation, school absenteeism, and mental health symptoms. Bivariate associations are presented in Table 4. Fewer worries about material reliability and changing were associated with fewer depression and anxiety symptoms. In contrast, positive perceptions of material, home and school environment needs were weakly associated with mental health.

More positive perceptions of materials, home and school environments predicted significantly higher odds of feeling confident to manage menstruation at home or school. Supporting item validity, positive school assessment was not associated with confidence at home. Material and home environments did show a weaker, but positive relationship with school management confidence, however this subscale includes items regarding menstrual materials and disposal which are likely to cross settings. Fewer concerns about material reliability, insecurity in changing and disposal access across contexts, and more positive perceptions of materials and home environments were associated with higher odds of attending school during menstruation. A higher MPNS total score, which captures girls' perceptions across all practices and environments, predicted much higher odds of confidence to manage menstruation and attending school during menses.

Table 4. Bivariate associations between scale scores and hypothesised correlates

\begin{tabular}{|c|c|c|c|c|c|c|}
\hline & & $\begin{array}{l}\text { Material and } \\
\text { home } \\
\text { environment }\end{array}$ & $\begin{array}{c}\text { Transport } \\
\text { and school } \\
\text { environment }\end{array}$ & $\begin{array}{l}\text { Material } \\
\text { reliability } \\
\text { concerns }\end{array}$ & $\begin{array}{c}\text { Change and } \\
\text { disposal } \\
\text { insecurity }\end{array}$ & Total \\
\hline & & $r(p)$ & $r(p)$ & $r(p)$ & $r(p)$ & $r(p)$ \\
\hline \multirow[t]{2}{*}{ DASS-21 total score ${ }^{1}(n=518)$} & & $\begin{array}{c}0.04 \\
(p=.333)\end{array}$ & $\begin{array}{c}0.16 \\
(p=<.001)\end{array}$ & $\begin{array}{c}-0.27 \\
(p<.001)\end{array}$ & $\begin{array}{c}-0.26 \\
(p<.001)\end{array}$ & $\begin{array}{c}-0.11 \\
(p=.013)\end{array}$ \\
\hline & $\%$ & OR $(95 \% \mathrm{Cl})$ & OR $(95 \% \mathrm{Cl})$ & OR $(95 \% \mathrm{Cl})$ & OR (95\%Cl) & OR $(95 \% \mathrm{Cl})$ \\
\hline $\begin{array}{l}\text { Felt confident to manage } \\
\text { menstruation at home }\end{array}$ & 80.92 & $\begin{array}{c}2.87 \\
(1.99,4.15)\end{array}$ & $\begin{array}{c}1.25 \\
(0.94,1.67)\end{array}$ & $\begin{array}{c}1.09 \\
(0.81,1.47)\end{array}$ & $\begin{array}{c}0.92 \\
(0.64,1.31)\end{array}$ & $\begin{array}{c}4.09 \\
(2.14,7.81)\end{array}$ \\
\hline $\begin{array}{l}\text { Felt confident to manage } \\
\text { menstruation at school }\end{array}$ & 51.72 & $\begin{array}{c}1.97 \\
(1.47,2.63)\end{array}$ & $\begin{array}{c}1.94 \\
(1.53,2.45)\end{array}$ & $\begin{array}{c}1.14 \\
(0.90,1.44)\end{array}$ & $\begin{array}{c}1.05 \\
(0.80,1.39)\end{array}$ & $\begin{array}{c}4.22 \\
(2.52,7.06) \\
\end{array}$ \\
\hline $\begin{array}{l}\text { Does not usually miss school during } \\
\text { menstruation }\end{array}$ & 61.90 & $\begin{array}{c}1.48 \\
(1.11,1.97)\end{array}$ & $\begin{array}{c}1.14 \\
(0.91,1.43)\end{array}$ & $\begin{array}{c}1.28 \\
(1.00,1.63)\end{array}$ & $\begin{array}{c}1.33 \\
(1.00,1.77)\end{array}$ & $\begin{array}{c}2.52 \\
(1.52,4.17)\end{array}$ \\
\hline $\begin{array}{l}\text { Did not miss school during last } \\
\text { menstrual period }\end{array}$ & 69.25 & $\begin{array}{c}1.11 \\
(0.82,1.50)\end{array}$ & $\begin{array}{c}0.99 \\
(0.78,1.26)\end{array}$ & $\begin{array}{c}1.54 \\
(1.19,2.00)\end{array}$ & $\begin{array}{c}2.10 \\
(1.51,2.91)\end{array}$ & $\begin{array}{c}2.62 \\
(1.52,4.50)\end{array}$ \\
\hline
\end{tabular}

${ }^{1}$ DASS score ranges from $0-42$, higher scores indicate greater anxiety and depression symptoms. ${ }^{2}$ not confident $=1.00$ 


\section{DISCUSSION}

The Menstrual Practice Needs Scale (MPNS-36) is a self-report measure to evaluate the extent to which an individuals' menstrual management practices and environments are perceived to meet their needs. Development was informed by past research, including review of qualitative and quantitative studies, and expert input. $[3,16,17,33]$ The final tool reflects experiences across a range of practices. Emergent factors were theoretically plausible and translated into interpretable subscales. The MPNS demonstrated good internal consistency, and acceptable test-retest reliability. Associations with hypothesised correlates supported the validity of the measure and its use in future research.

We hypothesised a-priori that emergent factors would reflect groups of practices, and that appraisals of environments would load on separate factors. Hypotheses were partially supported. The final four-and two-factor structure separated girls' appraisals of the reliability of their menstrual materials, home, and school environments. However, items capturing worries and concerns about changing environments, disposal and materials, loaded separately from ratings of comfort, satisfaction and adequacy of practices. These factors were not strongly correlated, or in the case of 'transport and school environment needs' and 'change and disposal insecurity', showed a small to modest negative correlation. Taken together, relationships suggest that greater satisfaction and comfort with menstrual practices does not translate into fewer worries about their reliability or risks to privacy or safety. Appraisals of privacy needs may be more strongly dictated to by internalised menstrual stigma, social relationships and norms, independent of the acceptability and comfort of other practices. Inspection of bivariate correlations suggested that trade-offs may be made between the favourability of the location to change menstrual materials and the accessibility of disposal options, contributing to negative subscale correlations. The use of 'worries' terminology in scale items was selected to best align with past qualitative reports and to prevent confusion which may arise in positively and negatively worded items using the same response options.[3,34] However, we acknowledge this may have been more likely to evoke anxieties than items asking about 'comfort' or having 'enough' of various resources. Feedback from enumerators suggested that girls in this study did not struggle with the nature of these items as the response options were in the affirmative direction for all questions. Enumerators did report that a measure included for validation, the Rosenberg Self-Esteem Scale,[35] which included positively and negatively worded items through use of alternate wording like "I do" versus "I do not" with the same response options caused difficulties for respondents. There was no such evidence of difficulties with reverse coded items in the MPNS-36 in enumerator feedback, frequencies, or visual inspection of surveys. Future research is needed to further investigate the interrelationships between menstrual needs, insecurities, and how females make menstrual practice decisions.

Measuring women's and girls' menstrual practice needs involves gaining an understanding of the acceptability, comfort, reliability of practices and insecurities around privacy, safety and exposure of menstrual status. Drawing on this theoretical underpinning, and the relatively acceptable performance of bi-factor and hierarchical models including a total score, we would argue that a total score capturing perceptions across the range of practice and environmental needs is appropriate. This score is likely to be of use to researchers and practitioners, summarising experience across the breadth of behaviours. Subscale and total scores were calculated using mean scores rather than factor scores. Mean scores allow for correction of single missing data points, by averaging across other items, and are accessible for practitioners who may not have access to the statistical packages needed to calculate factor scores. Since much of the data on menstrual experiences is collected as 
part of NGO monitoring and evaluation, comparability across these data and that in research studies is valuable so we suggest researchers use mean scores.

Insecurities about the privacy and safety of the locations used to change menstrual materials loaded on the same factor for questions concerning home and school environments. It is important to note that this indicates that these ratings co-varied, not that change locations in these settings were given the same ratings. School environments received much more negative appraisals, captured through frequencies and means. For research or practice evaluation that focuses on either home or school environments, the separate appraisal of location-specific subscales may need to be validated. However, further investigation is needed as covariation of home and school privacy ratings could suggest interdependencies between the two. It is plausible that experiences and learned expectations from home environments influence perceptions of school environments. Changes to individuals' expectations for their menstrual experience in response to interventions was an overarching theme of a recent meta-synthesis of qualitative studies of menstrual health interventions and would fit with this interpretation of our findings.[36] Alternatively, a joint predictor, such as internalised stigma, may contribute to both appraisals. This should be explored in future research and may indicate the need to assess both location responses even if interventions only focus on school infrastructure.

\section{Strengths and limitations}

Development of items drawing on the experiences of women and girls across low- and middleincome countries through systematic review indicates the potential for the MPNS-36 to be relevant across contexts. This approach was undertaken at the cost of specificity for the pilot population. A measure developed through qualitative study of the Soroti schoolgirl population may have yielded a different prioritisation of items. However, we were mindful of the ongoing measurement needs across contexts and calls for improved comparability, particularly across trial studies. $[5,8]$ At the same time, piloting and validation was undertaken in a single context and the measure should be evaluated in other languages and settings. Feedback from FGDs with enumerators in Niger, and online survey of experts suggest some languages or contexts may favour a 3-point response scale. Adapted response options as "less than half the time" and "more than half the time" may be more specific replacements for "sometimes" and "often" depending on the language of the scale. Our validation was limited by the lack of past quantitative research on predictive relationships between menstrual experience and outcomes, and the absence of other measures against which to assess convergent or divergent validity.

Some items asked of all respondents may not be applicable. For example, those who avoid school during menstruation were still asked about cleanliness, privacy and safety concerns and may report fewer worries as they manage their needs by avoiding changing materials at school. For simplicity, we recommend not using additional filters to questions, however response patterns should be explored in future studies and through cognitive interviewing, particularly where the measure is used in intervention studies.

As noted in methods, item reduction drew on factor analysis, but also considered the need for content validity through the coverage of different menstrual practices. We also prioritised brevity. Decisions to remove some items, such as those that were felt to duplicate practices may have reduced the internal consistency metrics of the scale but ensured items represented the breadth of practice experiences. Two subscales of three items each, 'material reliability concerns' and 'reuse insecurity' did not achieve acceptable internal consistency or test-retest reliability. This is likely due to the small number of items and variability within the short set. We retained these as separate 
subscales as we recognise concerns about the performance of menstrual materials and worries about exposure during washing and drying are salient parts of menstrual experiences.[3,12] Additional or refined items tested in future studies may improve the reliability of these subscales.

Test-retest reliability was assessed in a small sub-sample of participants. This sample size was reduced further due to the differential reliability between those reporting on the same menstrual period as their original survey. These data raise questions regarding the variability of menstrual experiences. Findings could also suggest that participating in the survey made girls more attentive to their needs during subsequent periods, leading to a change in their appraisals, a possibility that should be explored in subsequent studies and larger samples.

\section{Implications for research and practice}

Quantitative study of menstrual experiences has focused on measures of menstrual practices. Practices warrant investigation, however, increasingly menstrual health programming and policy have recognised that individuals and communities vary in their preferences and the practices viewed as preferable or acceptable.[37] The MPNS-36 prioritises participant perceptions of adequacy above researcher-defined 'adequate' menstrual practices. Although the definition of menstrual hygiene has evolved, the measure also provides an assessment of self-perceived menstrual hygiene status.

To date, research has relied on single practices, typically use of sanitary pads, to test associations between menstrual health and hypothesised consequences on school absenteeism or wellbeing. Such analyses fail to incorporate the range of practices needed for menstrual management, and poorly translate the findings from qualitative research into quantitative research questions. The MPNS-36 offers a way to test relationships between overarching menstrual practice experience and education, health, wellbeing and social participation consequences in cross-sectional or longitudinal studies. The measure could be applied in needs assessments or NGO monitoring and evaluation. The MPNS-36 could be used in trials of menstrual health interventions to assess how programs change practice experiences and would likely represent a key mediating assumption between interventions such as product provision or sanitation improvements, and end line impacts such as school attendance. Further studies will be needed to test the association between practice needs as measured through the MPNS-36 and school attendance, triangulating self-report data with more reliable methods such as school spot-checks.

Although the tested scale specified school as the location for a subset of items, this wording could be adapted to the workplace, or when 'away from home' when applied to adult or out-of-school samples. These groups require more attention,[3] and investigation of scale performance in these populations would be of value.

In sum, the menstrual practice needs scale is a self-report measure specifically developed to assess the extent to which an individuals' menstrual management practices and environments are perceived to meet their needs. The final instrument has high face validity, reflecting experiences across a range of practices and the total and subscale scores could be useful in needs assessment, monitoring and exploring intervention impact. 


\section{Ethical approvals}

All girls provided signed assent to participate. Parents were informed about the study through parent-teacher meetings at each school, teacher contact with parents, and information sheets in English and Ateso sent home with girls prior to the study. Parents were asked to contact the school or study staff if they did not consent to daughters' participation, or express concerns during parentteacher meetings. No parents expressed concerns about the study and no girls declined participation.

Ethical approval was provided by Johns Hopkins School of Public Health Institutional Review Board (IRB approval no: 00009073), and the Mildmay Uganda Research Ethics Committee (MUREC) (approval ref: 0212-2018). The Uganda National Council for Science and Technology (UNCST) approved the study (ref: SS279ES). Feedback on draft measure items by experts through online survey and focus group discussions of resident enumerators in Niger were exempted from ethical review by the Johns Hopkins School of Public Health Institutional Review Board. Participants of these consultations consented to participate.

\section{Acknowledgements}

We are most grateful to the participating girls and schools, and the dedicated team of research assistants who undertook data collection. We thank Dr Christian van Engers for developing the code for visual representation of the data in Supplementary Materials 2 and the administration of the www.menstrualpracticemeasures.org website. We are grateful to Prof. G.J. Melendez-Torres for his statistical guidance. We are indebted to the numerous experts in menstrual health who took the time to review draft items and provide their insights.

\section{Funding}

This study was funded by The Case for Her and the Osprey Foundation of Maryland.

\section{Contributions}

$\mathrm{JH}$ designed the study, undertook analysis, interpretation, and wrote the first draft of the manuscript. AN, CS, MR, KS, AA, contributed to study design, interpretation, and critically reviewed the manuscript. MR critically reviewed measure materials and analytic strategy. AN coordinated data collection and implemented study protocols. AA facilitated translation and back-translation of survey tools, supported data collection and feedback on the performance of items. All authors have approved the final manuscript.

\section{Data sharing statement}

Deidentified data is available at: https://osf.io/qshkc/. The final MPNS-36 measure and scoring information is available online from www.menstrualpracticemeasures.org

\section{Competing interests}

Calum Smith works for Irise International, an organisation dedicated to creating a world where all women and girls can reach their full potential, regardless of their periods. Irise International receives funding from various sources to develop school-based menstrual health interventions in East Africa and from Sustain for Life to work with schools in Soroti, Uganda. Agnes Nansubuga and Agnes Akullo work for Irise Institute East Africa, a local implementing partner of Irise International.

$J H, M R, K J S$ declare no competing interests. 


\section{REFERENCES}

1. Bobel C. Making Menstruation Matter in the Global South: Mapping a Critical History. The Managed Body: Springer; 2019. p. 69-109.

2. Sommer M, Caruso BA, Sahin M, Calderon T, Cavill S, Mahon T, et al. A Time for Global Action: Addressing Girls? Menstrual Hygiene Management Needs in Schools. PLoS Med. 2016;13(2):e1001962.

3. Hennegan J, Shannon AK, Rubli J, Schwab KJ, Melendez-Torres GJ. Women's and girls' experiences of menstruation in low- and middle-income countries: a systematic review and qualitative metasynthesis. PLOS Medicine. 2019;16(5):e1002803.

4. Tellier S, Hyttel M. Menstrual Health Management in East and Southern Africa: a Review Paper. South Africa: UNFPA; 2018.

5. Hennegan J, Montgomery P. Do Menstrual Hygiene Management Interventions Improve Education and Psychosocial Outcomes for Women and Girls in Low and Middle Income Countries? A Systematic Review. PloS one. 2016;11(2):e0146985.

6. Phillips-Howard PA, Caruso B, Torondel B, Zulaika G, Sahin M, Sommer M. Menstrual hygiene management among adolescent schoolgirls in low-and middle-income countries: research priorities. Global health action. 2016;9(1):33032.

7. Hennegan J. Menstrual hygiene management and human rights: the case for an evidence-based approach. Women's Reproductive Health. 2017;4(3):212-31.

8. UNICEF. Guidance on Menstrual Health and Hygiene. New York, USA: UNICEF. Available from https://www.unicef.org/wash/files/UNICEF-Guidance-menstrual-health-hygiene-2019.pdf [Accessed July 2019]; 2019.

9. Sommer M, Sahin M. Overcoming the Taboo: Advancing the Global Agenda for Menstrual Hygiene Management for Schoolgirls. American journal of public health. 2013;103(9):1556-9.

10. Crichton J, Okal J, Kabiru CW, Zulu EM. Emotional and Psychosocial Aspects of Menstrual Poverty in Resource-Poor Settings: A Qualitative Study of the Experiences of Adolescent Girls in an Informal Settlement in Nairobi. Health Care for Women International. 2013;34(10):891-916.

11. House S, Cavill S, Mahon T, Hunger AA, UNICEF, Advocates W. Menstrual hygiene matters: A resource for improving menstrual hygiene around the world: WaterAid; 2012.

12. MacRae ER, Clasen T, Dasmohapatra M, Caruso BA. 'It's like a burden on the head': Redefining adequate menstrual hygiene management throughout women's varied life stages in Odisha, India. PLOS ONE. 2019;14(8):e0220114.

13. Sommer M, Figueroa C, Kwauk C, Jones M, Fyles N. Attention to menstrual hygiene management in schools: An analysis of education policy documents in low-and middle-income countries. International Journal of Educational Development. 2017;57:73-82.

14. United Nations Children's Fund (UNICEF), World Health Organization. Drinking Water, Sanitation and Hygiene in Schools: Global Baseline Report 2018. New York: United Nations Children's Fund (UNICEF) and World Health Organization; 2018.

15. Torondel B, Sinha S, Mohanty JR, Swain T, Sahoo P, Panda B, et al. Association between unhygienic menstrual management practices and prevalence of lower reproductive tract infections: a hospital-based cross-sectional study in Odisha, India. BMC infectious diseases. 2018;18(1):473.

16. Hennegan JM, Dolan C, Wu M, Scott L, Montgomery P. Measuring the prevalence and impact of poor menstrual hygiene management: a quantitative survey of schoolgirls in rural Uganda. BMJ Open. 2016;6(12):e012596.

17. Hennegan J, Sol L. Confidence to manage menstruation at home and at school: findings from a crosssectional survey of schoolgirls in rural Bangladesh. Culture, health \& sexuality. 2019:1-20.

18. Zimmerman L, Olson H, Tsui A, Radloff S. PMA2020: Rapid Turn-Around Survey Data to Monitor Family Planning Service and Practice in Ten Countries. Studies in family planning. 2017;48(3):293-303. 
19. Khan SM, Bain RE, Lunze K, Unalan T, Beshanski-Pedersen B, Slaymaker T, et al. Optimizing household survey methods to monitor the Sustainable Development Goals targets 6.1 and 6.2 on drinking water, sanitation and hygiene: A mixed-methods field-test in Belize. PloS one. 2017;12(12):e0189089.

20. Caruso BA, Clasen T, Yount KM, Cooper HL, Hadley C, Haardörfer R. Assessing women's negative sanitation experiences and concerns: the development of a novel sanitation insecurity measure. International Journal of Environmental Research and Public Health. 2017;14(7):755.

21. UNICEF, Columbia University. 'MHM in Ten': Advancing the MHM Agenda in WASH in Schools. New York: Columbia University; 2014. https://www.mhmvirtualconference.com/mhm-in-ten

22. Uganda Bureau of Statistics (UBOS), ICF. Uganda Demographic and Health Survey 2016. Kampala, Uganda and Rockville, Maryland: UBOS and ICF.; 2018.

23. Afrobarometer. Surveys and methods: Afrobarometer; 2018. Available from https://afrobarometer.org [Accessed September 2018]

24. Lovibond SH, Lovibond PF. Manual for the depression anxiety stress scales. Psychology Foundation of Australia. 1996.

25. Rubin D. Multiple Imputation for Nonresponse in Surveys: Wiley; 1987.

26. Enders CK, Mansolf M. Assessing the fit of structural equation models with multiply imputed data. Psychological methods. 2018;23(1):76.

27. Boateng GO, Neilands TB, Frongillo EA, Melgar-Quiñonez HR, Young SL. Best practices for developing and validating scales for health, social, and behavioral research: a primer. Frontiers in public health. 2018;6.

28. Svetina D, Rutkowski L, Rutkowski D. Multiple-Group Invariance with Categorical Outcomes Using Updated Guidelines: An Illustration Using M plus and the lavaan/semTools Packages. Structural Equation Modeling: A Multidisciplinary Journal. 2019:1-20.

29. Wu H, Estabrook R. Identification of confirmatory factor analysis models of different levels of invariance for ordered categorical outcomes. Psychometrika. 2016;81(4):1014-45.

30. Gadermann AM, Guhn M, Zumbo BD. Estimating ordinal reliability for Likert-type and ordinal item response data: a conceptual, empirical, and practical guide. Practical Assessment, Research \& Evaluation. 2012;17(3).

31. Koo TK, Li MY. A guideline of selecting and reporting intraclass correlation coefficients for reliability research. Journal of chiropractic medicine. 2016;15(2):155-63.

32. Carpenter S. Ten steps in scale development and reporting: A guide for researchers. Communication Methods and Measures. 2018;12(1):25-44.

33. Hennegan J, Zimmerman L, Shannon AK, Exum NG, OlaOlorun F, Omoluabi E, et al. The relationship between household sanitation and women's experience of menstrual hygiene: Findings from a cross-sectional survey in Kaduna State, Nigeria. International journal of environmental research and public health. 2018;15(5).

34. Sclar G, Penakalapati G, Caruso B, Rehfuess EA, Garn J, Alexander K, et al. Exploring the relationship between sanitation and mental and social well-being: A systematic review and qualitative synthesis. Social Science \& Medicine. 2018.

35. Rosenberg M. Rosenberg self-esteem scale (SES). Society and the adolescent self-image. 1965.

36. Shannon AK, Melendez-Torres GJ, Hennegan J. How do women and girls experience menstrual health interventions in low- and middle-income countries? Insights from a systematic review and qualitative metasynthesis. Under Review.

37. UNICEF. Guide to menstrual hygiene materials. New York, USA: UNICEF; 2019. Available from: https://www.unicef.org/wash/files/UNICEF-Guide-menstrual-hygiene-materials-2019.pdf [Accessed June 2019]. 